\title{
Sonodynamic and Photodynamic Therapy in Advanced Pancreas Carcinoma - A Case Report
}

\author{
Wenying Zhang ${ }^{1}$, Kun Li ${ }^{1}$, Lucy Qing Li ${ }^{2 *}$, Xiaohuai Wang ${ }^{3}$, Xie Bo ${ }^{3}$ and Hong Xu${ }^{2}$ \\ ${ }^{1}$ Oncology Department, Guangzhou Medical University of Chinese Medicine Affiliated Jinshazhou Hospital, China \\ ${ }^{2}$ College of Engineering and Science, Victoria University, Australia \\ ${ }^{3}$ Department of Oncology, Southern Theater, General Hospital of the People's Liberation Army of China
}

*Corresponding author: Lucy Qing Li, College of Engineering and Science, Victoria University, P0 Box 14428, Melbourne 8001, Victoria, Australia.

Received Date: January 10, 2020

Published Date: January 27, 2020

\begin{abstract}
Photodynamic Therapy (PDT) is a therapeutic treatment for certain kinds of tumors, approved by the FDA in 1998. Recent research data shows that a related procedure, Sonodynamic Therapy (SDT), is another new promising complementary method for cancer treatment. Here we report clinical results in an advanced pancreas cancer patient who was treated using a combination of Sonodynamic and Photodynamic Therapy (SPDT), along with conventional therapies. The patient was pathologically diagnosed with metastatic pancreas carcinoma (non-respectable advanced pancreas cancer) by the patient's local oncology team. The advanced pancreas carcinoma had responded unsatisfactorily to conventional therapies. SPDT treatment involved using a new sensitizing agent Sonoflora a TM (SFa), which was administered sublingually; 12-hour latter, the patient was treated with a combination of light and ultrasound devices, and a low dosage of chemotherapy simultaneously as a whole body treatment (SPDT follows the chemotherapy cycle periodically). Patient had good partial responses. The results indicate that SPDT could be a promising new complementary therapeutic combination for the treatment of pancreas cancer.
\end{abstract}

Keywords: Sonodynimic therapy; Photodynamic therapy; Pancreas cancer; Chemotherapy; HIFU sensitizer; Sonosensitizer

\section{Introduction}

Photodynamic Therapy (PDT) was first approved as established therapeutic method by the FDA for certain kinds of cancer in 1998. However, In view of the limitation of the penetration of light energy into tumor tissues [1,2], the successful application from LED or coherent laser emission source of PDT has been mostly limited to superficial pathology or through invasive method which could approach to the pathology with unwanted side- effects. Ultrasound is a mechanical wave which could penetrate tissue excellently and safely without major attenuation of its energy $[3,4]$. Therefore, the potential medical application of ultrasound has been evaluated extensively and has led to the routine use of ultrasound for diagnostic imaging. Sonodynamic Therapy (SDT) has been widely researched to develop as a complementary or alternative therapy to PDT [5-10]. In SDT treatment, patients first ingest a sonosensitizer, an agent that can be activated by ultrasound. This agent is tailored to be activated through the use of low-intensity ultrasound energy producing a cascade of endogenous cytotoxic radicals. The therapy is thus similar to PDT, which is an attractive modality for cancer treatment with potential to focus the energy on tumor sites buried deep in tissues and to locally activate a preloaded sonosensitizer. The body transmits ultrasound energy much more efficiently than light energy, and this is a critical advantage when treating inside tumors and with minimal damage to peripheral healthy tissue. So far, many compounds were found to have sonodynamic activity [513]. In recent years, a novel sonosensitizer was developed by the late Donald Burke, MD, of Advanced Technologies, Boston, USA. He named this Sonoflora $1^{\mathrm{TM}}$ (SF1). This agent is a chlorophyll derivative with very high sonodynamic as well as photodynamic activity. Embryonic zebra fish assay had showed SF1 has no evidence of toxicity [3]. Our animal studies demonstrate that SDT with SF1 inhibits the growth of mouse S-180 sarcoma, even when the tumor is covered by a bone [4]. We had successfully used Sonodynamic and Photodynamic Therapy (SPDT) with SF1 and a portable ultrasound device in terminally ill breast cancer patients [14]. 
Here, we report initial clinical data using two new chlorophyllderived sono-photo-sensitizing agents (SFa and UF) along with a new equipment, supplied by EEC Biotech (Guangzhou) Co. for systemic SDT. The sensitizers SFa and UF were given to patients through lingual absorption on day one and day three. On Day two today four ozone treatment was given twice a day. The tumor area and the whole body were irradiated by ultrasound on day two and day four twice a day for 40 mins each time, then by red LED light at $45 \mathrm{mV} / \mathrm{cm} 2$ and $554 \mathrm{~nm}$ of wavelength for 30 minutes once a day. We used multiple ultrasound transducers within systemic SDT device, the tumor area and whole body was irradiated for 40 minutes at $75 \%$ pulse, $1 \mathrm{MHz}$ and $2.0 \mathrm{~W} / \mathrm{cm} 2$. The second week the same treatment was repeated. One cycle of SPDT included two round treatments and a week free.

\section{Case Presentation}

The patient is female, at the age of 61 . She went to the local hospital for medical examination for abdominal pain with jaundice for a month in November 2017. Examination indicated space occupying lesion in pancreatic head. CA19-9 was high. Local Doctor diagnosed pancreatic cancer. A stand was implanted in patient's bile duct. Patient underwent chemotherapy of Gemcitabine for three months after her jaundice was gone. Patient then had severe digestive tract symptoms, bone marrow suppression grade IV, Rechecked CA199 dropped significantly. However, no tumor shrinkage was seen in original lesion in CT images. Patient had been on Abraxane+Gemcitabine for three months Since February 2018, during which she was showed digestive tract symptoms and had bone marrow suppression as well. Rechecked CT scan showed progression and patient started to build up a large amount of ascites.

Surgery and radiation were not suitable for the patients as metastasis and location of the tumor in pancreatic head. Two types of chemo failed after three months treatment and no conventional therapy was available for option.

July 2018 after all the necessary tests, the patient came to our hospital for treatment, she started the first cycle of SPDT on July 25, 2018, cooperating chemotherapy with targeted medicine Erlotinib 150mg Po Qd +Xeloda 1.5g Po Bid D1-14+Oxaliplatin 80mg D3, $50 \mathrm{mg}$ D10, assisting with 5 days of HIFU treatments. During the course, patient had Grade II of digestive symptoms, nausea and vomiting, which were attenuated after symptomatic treatment. Ascites reduced obviously after the first cycle treatment. Tumor marker dropped from $4289 \mathrm{U} / \mathrm{ml}$ to $3038 \mathrm{U} / \mathrm{ml}$.

Second cycle of SPDT cooperating chemotherapy (same regimen as the first one). During this course, patient had mild nausea, no myelosuppression. Tumor marker CA199 after second cycle of SPDT dropped to $1873 / \mathrm{ml}$ and ascites reduced rapidly. Doctor's assessment indicated "stable".

Third and fourth cycles of SPDT started on September 05, 2018, continuing chemotherapy with Erlotinib 150mg Po Qd +Xeloda 1.0g in the morning and $1.5 \mathrm{~g}$ in the afternoon D1-14+0xaliplatin $80 \mathrm{mg}$ D3. Reduced the dosage of chemo's for her Grade III nausea, with Grade I myelosuppression.

A review of PET/CT (Oct 15, 2018) compared with July 23, 2018 before treatment.

A. Metabolism in the head of pancreas slightly rises, size of focal obviously narrows and the metabolism obviously reduced. Considered suppressed in most tumor activity after treatment (Figure 1).

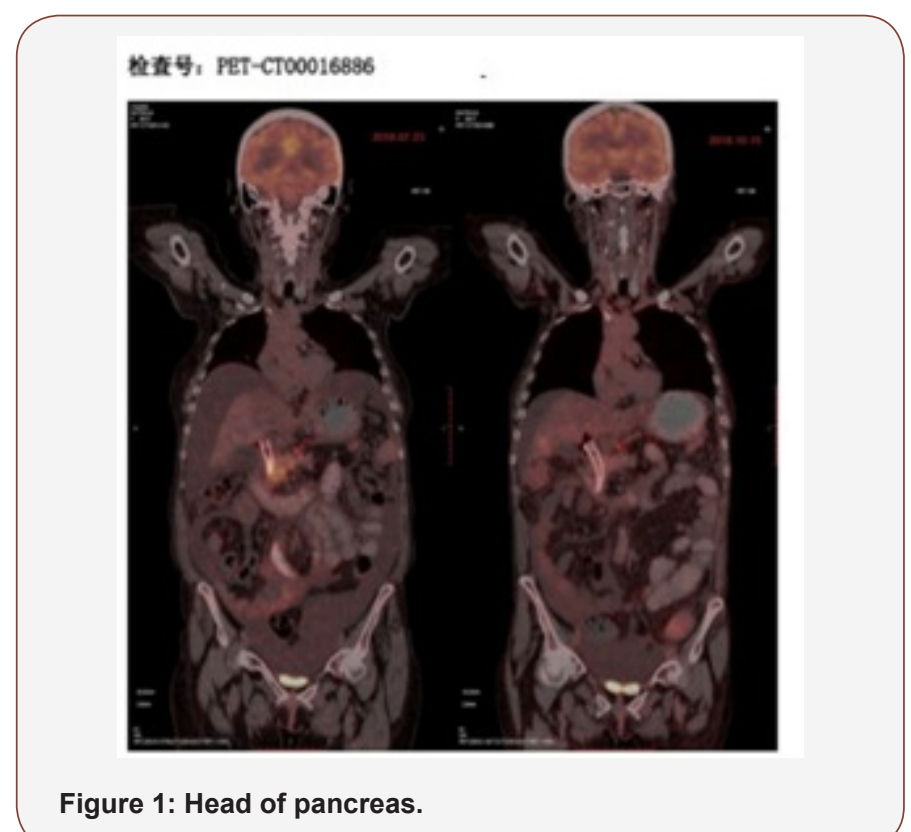

B. Multiplelymph nodes around pancreas and retroperitoneal region, multiple metastatic tumor in liver, metabolism reduced compared with the former scan. Considered suppressed of activity after treatment (Figure 2).

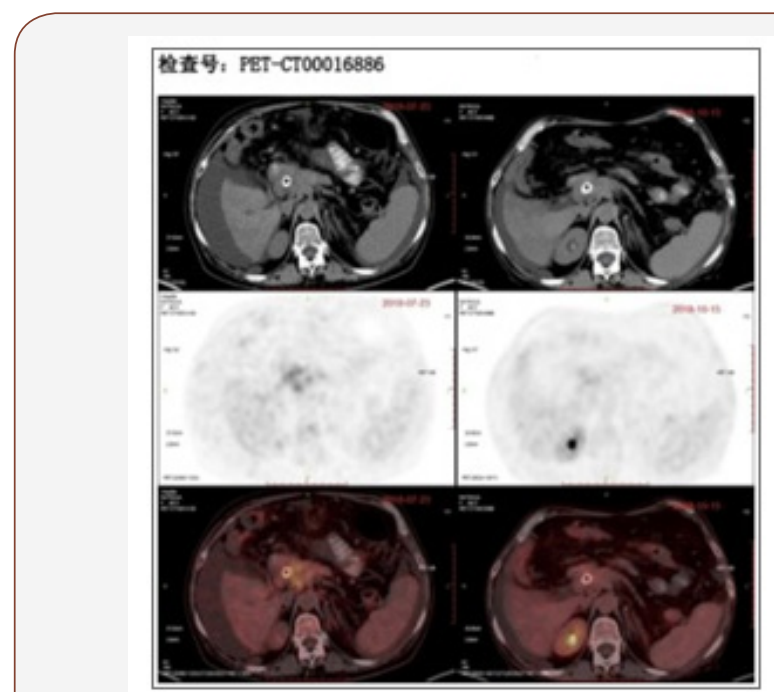

Figure 2: Primary tumor in pancreas.

C. The fluid collection in pelvic and abdominal cavity reduced obviously (Figures 3\&4). 


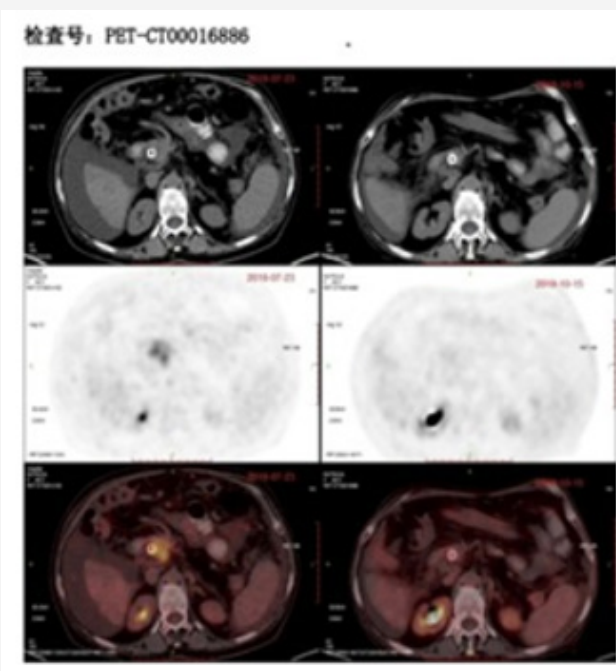

Figure 3: Fluid in pelvic and abdominal cavity.

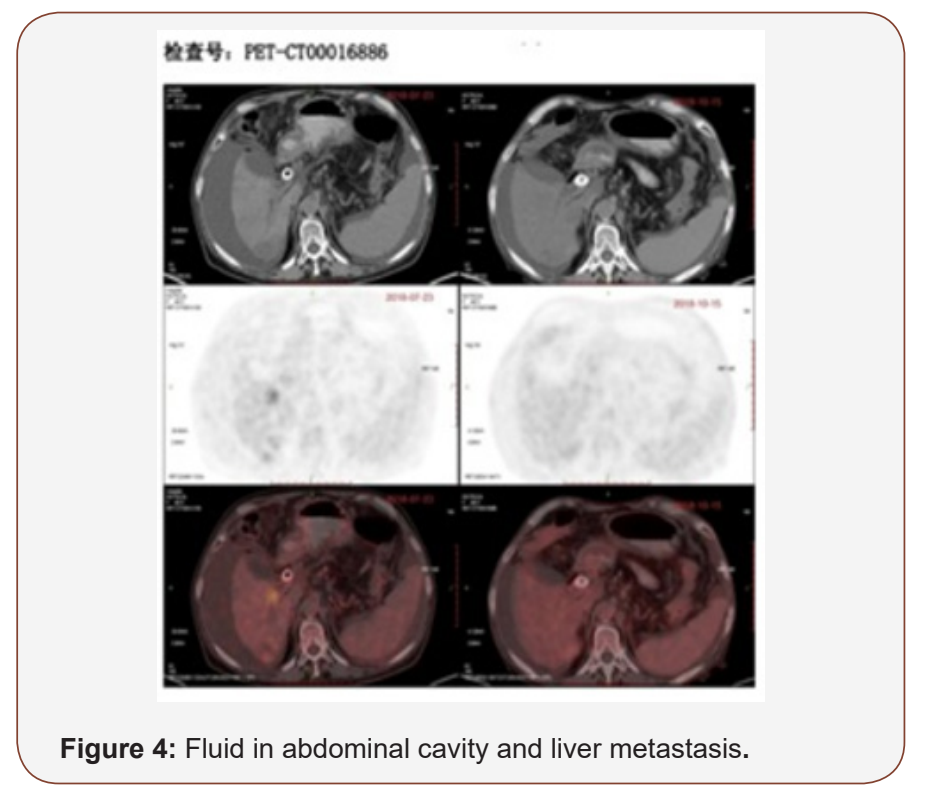

\section{Discussion}

SPDT protocol shows a potential promise over many years as a safe and well tolerated non-invasive treatment in advanced metastatic cancer. No adverse events were noted following administration of SFa and UF. The case did not have photosensitive dermatitis or rash. We used photo-diagnostic method with SFa and UF, were found that the sensitizer's specific fluorescence was only seen on superficial tumor, nothing on the normal skin, which implying our sensitizers did selectively accumulate in tumor. Compared with conventional PDT, such as Photofrin II-PDT, SPDT had almost no photo-dermatitis, when we tried to irradiate with laser light locally at $300 \mathrm{~J} / \mathrm{cm} 2$ energy 24 hours after taken the sensitizers. This further indicated the sensitizer's high clearance from normal tissues. The experiment carried indicated the sensitizer eliminate from the normal organs really quick even in less than $12 \mathrm{~h}$, that is why we could apply the systemic SDT after 12 hours administration of sensitizer. The new systemic SDT device has 153 ultrasonic heads on the bottom and upper ultrasonic device. Under computer control, every ultrasound head simultaneously emitted same ultrasound at $75 \%$ pulse, $1 \mathrm{MHz}$, and $2.0 \mathrm{~W} / \mathrm{cm} 2$ for 40 minutes twice a day for 2 days during the protocol. This may indicate ultrasounds used in this study may be strong enough to activate the sensitizer in primary or secondary tumor masses in whole body.

In this case, SPDT with SFa and UF was well tolerated. Unlike chemotherapy and radiation therapy, SPDT has almost no toxic effects, nor has it affected marrow and gastrointestinal function, or affect the important organs such as heart, lungs, liver, or kidney. Patient had metastases in the liver before the treatment, her ascites built up for large amount and her Performance Status (PS) score was 3 , her PS score was 2 . She could spend more time outside of her room. Her ascites, swollen legs and feet were improving remarkably. She could tolerate the chemotherapy well with grade 1 adverse effect of the gastrointestinal reaction, and her liver and kidney function blood test showed no obvious changes after the combined therapies.

Pain in the tumor regions was the main side effect of SPDT and HIFU. Some patients have no pain in their liver but patients may feel the pain in the ultrasound bath tube. If the patient kept still in the ultrasound bath, the energy could accumulate in the deep organs, especially in the bone, which causes pain. The problem can be avoided by using pulsed ultrasound or remind patients slowly move in the water. SPDT-induced tumor breakdown can also induce local pain and make patients feel weak and tired, in our experience patients could recover soon in a few days. HIFU could also cause pain in the treatment area but this pain could be released in hours. HIFU could also help quick relief the pancreas cancer patients' back pain after several times of HIFU therapy.

High intensity focused ultrasound which is a physical therapy has developed during the past 20 years [15]. It uses the ultrasonic waves with the function of penetration and focusing capability to form a high-calorie focal field on the tumor location where the depth less than $9 \mathrm{~cm}$. It makes the solid tumor protein coagulation and necrosis, then kill the target cells. At present, high intensity focused ultrasound has been widely used in the treatment of solid tumors. In recent years, high intensity focused ultrasound in the treatment of liver cancer has attracted more and more attention. The application of high intensity focused ultrasound become more and more common as a local palliative treatment of tumor, it has the advantages on safety, effectiveness and less side effects. We adopt the mature ultrasonic focusing knife combined with the new generation of sensitivity agent which can achieve better clinical efficacy in treating local tumor without systemic side effects. We found that the tumor has been shrunk obviously and metabolic activity has dropped after the combined treatment.

Possible cytotoxic mechanisms may include generation of sonosensitizer-derived radicals which initiates chain peroxidation of membrane lipids via peroxyl and/or alkoxyl radicals, the physical destabilization of the cell membrane by the sonosensitizer thereby rendering the cell more susceptible to shear forces and cavitation effects. Especially we administered the chemotherapy with the 
SPDT simultaneously, ultrasound may enhance drug transport across the cell membrane [11,16,17].

In summary, SPDT with SFa and UF was well tolerated and had demonstrated significant therapeutic benefits for the patient with advanced pancreas cancer. There also appears to be a potential role for SPDT in adjuvant pancreas cancer treatment, considering that pancreas cancer has a low survival rate. There are some new targeted medicines and immunotherapies which have been used for other solid tumors but have not been used promisingly for pancreas cancer. SPDT may have further benefits which await further investigation.

\section{Acknowledgement}

None.

\section{Conflict of Interest}

No conflict of interest. EEC is a supplier for the Sfa and the ultrasound equipment, no financial involvement in such clinical practice.

\section{References}

1. Dolmans DE, Fukumura D, Jain RK (2003) Photodynamic therapy for cancer. Nat Rev Cancer 3(5): 380-387.

2. Brancaleon L, Moseley H (2002) Laser and non-laser light sources for photodynamic therapy. Lasers Med Sci 17(3): 173-186.

3. Lewis TJ (2010) Toxicity and cytopathogenic properties toward human melanoma cells of activated cancer therapeutics in Zebra fish. Integr Cancer Ther 9(1): 84-92.

4. Xiaohuai Wang, Lewis TJ, Mitchell D (2008) The tumoricidal effect of sonodynamic therapy (SDT) on S-180 sarcoma in mice. Integr Cancer Ther 7(2): 96-102.

5. Kinoshita M, Hynynen K (2006) Mechanism of porphyrin-induced sonodynamic effect: possible role of hyperthermia. Radiat Res 165(3): 299-306.
6. Yumita N, Umemura S (2004) Sonodynamic anti tumour effect of chloroaluminum phthalocyanine tetra sulfonate on murine solid tumour. J Pharm Pharmacol 56(1): 85-90.

7. Huang D, Okada K, Komori C, Itoi E, Kawamura K, et al. (2004) Ultrastructure of sarcoma 180 cells after ultrasound irradiation in the presence of sparfloxacin. Anticancer Res 24(3a): 1553-1559.

8. Yumita N, Han QS, Kitazumi I, Umemura S (2008) Sonodynamicallyinduced apoptosis, necrosis, and active oxygen generation by mono-laspartyl chlorin e6. Cancer Sci 99(1): 166-172.

9. Yumita N, Iwase Y, Nishi K, Ikeda T, Komatsu H, et al. (2011) Sonodynamically-induced antitumor effect of mono-l-aspartylchlorine6 (NPe6). Anticancer Res 31(2): 501-506.

10. Shibaguchi H, Tsuru H, Kuroki M, Kuroki M (2011) Sonodynamic cancer therapy: a non-invasive and repeatable approach using low-intensity ultrasound with a sonosensitizer. Anticancer Res 31(7): 2425-2429.

11. Kondo T, Kano E (1988) Effect of free radicals induced by ultrasonic cavitation on cell killing. Int J Radiat Biol 54(3): 475-486.

12. Kondo T, Umemura S, Tanabe K (2000) Novel therapeutic applications of ultrasound: utilization of thermal and cavitational effects. J pn J Hyperthermic Oncol 16: 203-216.

13. Feril LB Jr, Kondo T (2004) Biological effects of low intensity ultrasound: The mechanism involved, and its implications on therapy and on biosafety of ultrasound. J Radiat Res 45(4): 479-489.

14. Wang X, Zhang W, Xu Z, Luo Y, Mitchell D, et al. (2009) Sonodynamic and Photodynamic Therapy in Advanced Breast Carcinoma: A report of Three Cases. Integr Cancer Ther 8(3): 283-287.

15. Meyers PA, Schwartz CL, Krailo MD, Healey JH, Bernstein ML, et al. (2008) Osteosarcoma: the addition of muramyl tripeptide to chemotherapy improves overall survival - a report from the Children's Oncology Group. J Clin Oncol 26(4): 633-638.

16. Rosenthal I, Sostaric JZ, Riesz P (2004) Sonodynamic therapy--a review of the synergistic effects of drugs and ultrasound. Ultrason Sonochem 11(6): 349-363.

17. Wakako H, Hidemi H, Loreto B, Feril J, Nobuki K, et al. (2006) Comparison between sonodynamic effect and photodynamic effect with photosensitizers on free radical formation and cell killing. Ultrason Sonochem 13(6): 535-542. 